\title{
Mehler hemigroups and embedding of discrete skew convolution semigroups on simply connected nilpotent \\ Lie groups
}

Peter Becker-Kern and Wilfried Hazod

Preprint 2008-10

Mai 2008

Fakultät für Mathematik

Technische Universität Dortmund

Vogelpothsweg 87

44227 Dortmund

$\underline{\text { tu-dortmund.de/MathPreprints }}$ 



\title{
MEHLER HEMIGROUPS AND EMBEDDING OF DISCRETE SKEW CONVOLUTION SEMIGROUPS ON SIMPLY CONNECTED NILPOTENT LIE GROUPS
}

\author{
PETER BECKER-KERN AND WILFRIED HAZOD
}

\begin{abstract}
It is shown how discrete skew convolution semigroups of probability measures on a simply connected nilpotent Lie group can be embedded into Lipschitz continuous semistable hemigroups by means of their generating functionals. These hemigroups are the distributions of increments of additive semi-selfsimilar processes. Considering these on an enlarged space-time group, we obtain Mehler hemigroups corresponding to periodically stationary processes of Ornstein-Uhlenbeck type, driven by certain additive processes with periodically stationary increments. The background driving processes are further represented by generalized Lie-Trotter formulas for convolutions, corresponding to a random integral approach known for finite-dimensional vector spaces.
\end{abstract}

\section{INTRODUCTION}

In the last decades there has been considerable interest in selfsimilar stochastic processes obeying certain space-time scaling properties. These processes are useful to model a wide variety of scaling phenomenas in diverse fields. Our focus is on additive processes, additionally assuming independent increments. In this case the family of distributions of the increments builds a stable hemigroup of probability measures. By Lamperti's [17] transformation the processes are closely connected with stationary Ornstein-Uhlenbeck type processes. On $\mathbb{R}^{d}$ a selfsimilar additive process can be represented by random integrals with respect to a background driving Lévy process and this representation extends to the Ornstein-Uhlenbeck process; see [14]. On groups such integral representations are not available, but there exist weak representations by Lie-Trotter formulas for convolutions on an enlarged space-time group; see $[8,9]$. The resulting objects on groups are a convolution semigroup corresponding to the background driving Lévy process and a Mehler semigroup corresponding to the Ornstein-Uhlenbeck process. There has also been drawn attention to Mehler semigroups as Markovian transition operators on infinite dimensional vector spaces and its interplay to Ornstein-Uhlenbeck processes and skew convolution semigroups; see

2000 Mathematics Subject Classification. Primary 60B15; Secondary 60G18, 60G51, 43A05.

Key words and phrases. Lipschitz continuous hemigroup, semi-selfsimilar additive process, spacetime group, periodic Ornstein-Uhlenbeck process, background driving process, generalized LieTrotter formula. 
$[3,4,5,7,18,22]$. A skew convolution semigroup arises as the family of cofactors for the selfdecomposable one-dimensional marginal distributions of a selfsimilar additive process. A random integral representation for operator-selfdecomposable measures has already been obtained by Jurek and Vervaat [15]. Conversely, given a skew convolution semigroup it is possible to reconstruct the stable hemigroup and thus all other distributional families mentioned above; see [8].

Our aim is to generalize these results for additive processes with the weaker scaling property of semi-selfsimilarity on a discrete scale. We focus on the question of reconstructing a semi-stable hemigroup (distributions of the increments) and other objects from a discrete skew convolution semigroup on a locally compact group $\mathbb{G}$. To motivate our studies, we first survey on results in this respect for operator-semi-selfsimilar processes on $\mathbb{R}^{d}$.

Let $\left\{X_{t}\right\}_{t \geq 0}$ be an additive stochastic process on $\mathbb{R}^{d}$, i.e. $X_{0}=0, t \mapsto P_{X_{t}}$ is weakly continuous and $\left\{X_{t}\right\}_{t \geq 0}$ has independent increments. Let $Q \in \mathrm{GL}\left(\mathbb{R}^{d}\right)$ be such that $e^{-t Q} \rightarrow 0$ as $t \rightarrow \infty$. The additive process $\left\{X_{t}\right\}_{t \geq 0}$ is called operatorsemi-selfsimilar with exponent $Q$ if $\left\{c^{Q} X_{t}\right\}_{t \geq 0}=\left\{X_{c t}\right\}_{t \geq 0}$ for some $c>1$ in the sense of equality of all finite-dimensional distributions. Due to the construction of random integrals in [15] the processes $\left\{Y_{t}^{(+)}\right\}_{t \geq 0}$ and $\left\{Y_{t}^{(-)}\right\}_{t \geq 0}$ defined by

$$
Y_{t}^{(+)}=\int_{1}^{e^{t}} s^{-Q} d X_{s} \quad \text { and } \quad Y_{t}^{(-)}=\int_{e^{-t}}^{1} s^{Q} d X_{s}
$$

are i.i.d. additive processes with $\log c$-stationary increments, i.e. $Y_{t+\log c}^{( \pm)}-Y_{s+\log c}^{( \pm)}$is equal in distribution to $Y_{t}^{( \pm)}-Y_{s}^{( \pm)}$for all $0 \leq s \leq t$, and with a certain finite logarithmic moment condition, from which the operator-semi-selfsimilar process can be almost surely pathwise recovered by

$$
X_{t}= \begin{cases}\int_{-\log t}^{\infty} e^{-s Q} d Y_{s}^{(-)} & \text {if } 0 \leq t \leq 1, \\ X_{1}+\int_{0}^{\log t} e^{s Q} d Y_{s}^{(+)} & \text {if } t>1 .\end{cases}
$$

The process $\left\{Y_{t}^{( \pm)}\right\}_{t \geq 0}$ is called the background driving additive periodic process. For details see $[2,20]$. Conversely, any additive process with $\log c$-stationary increments and with certain finite logarithmic moment defines an additive operatorsemi-selfsimilar process in this way. The random integral representation (1.1) easily carries over to Ornstein-Uhlenbeck type processes $\left\{U_{t}^{(+)}=e^{-t Q} X_{e^{t}}\right\}_{t \geq 0}$ and $\left\{U_{t}^{(-)}=e^{t Q} X_{e^{-t}}\right\}_{t \geq 0}$ given by Lamperti's [17] transformation. These processes are periodically stationary Markov processes with period $\log c$, i.e. $\left\{U_{t+\log c}^{( \pm)}\right\}_{t \geq 0}=\left\{U_{t}^{( \pm)}\right\}_{t \geq 0}$ again in the sense of equality of all finite-dimensional distributions. Their Markov transition operators $P_{s, t}(f)(x)=\mathbb{E}\left(f\left(U_{t}^{( \pm)}\right) \mid U_{s}^{( \pm)}=x\right)$ for $0 \leq s \leq t$ and bounded measurable $f: \mathbb{R}^{d} \rightarrow \mathbb{R}$ can easily be shown to be $\log c$-periodic Feller hemigroups, 
i.e. for $0 \leq s \leq r \leq t$ we have $P_{s, r} P_{r, t}=P_{s, t}, P_{s+\log c, t+\log c}=P_{s, t}$, and $P_{s, t}(f) \in \mathcal{C}_{b}\left(\mathbb{R}^{d}\right)$ for every $f \in \mathcal{C}_{b}\left(\mathbb{R}^{d}\right)$, which we call Mehler hemigroups in analogy to Mehler semigroups for stationary Ornstein-Uhlenbeck processes.

Turning back to the operator-semi-selfsimilar additive process $\left\{X_{t}\right\}_{t \geq 0}$, the family of distributions of the increments $\left(\mu_{s, t}=P_{X_{t}-X_{s}}\right)_{0 \leq s \leq t}$ builds a continuous semistable convolution hemigroup, i.e. for $0 \leq s \leq r \leq t$ we have $\mu_{s, r} * \mu_{r, t}=\mu_{s, t}$, $c^{Q} \mu_{s, t}=\mu_{c s, c t}$, and $(s, t) \mapsto \mu_{s, t}$ is weakly continuous. Especially, $\mu=\mu_{0,1}$ is operator-semi-selfdecomposable, i.e. for $n \in \mathbb{N}$ we have $\mu=c^{-n Q} \mu * \nu_{n}$ for some cofactors $\left(\nu_{n}\right)_{n \in \mathbb{N}} \subseteq \mathcal{M}^{1}\left(\mathbb{R}^{d}\right)$, namely $\nu_{n}=\mu_{c^{-n}, 1}$. The cofactors build a discrete skew convoluton semigroup, i.e. $\nu_{n+m}=\nu_{n} * c^{-n Q} \nu_{m}$ for all $n, m \in \mathbb{N}$, and we further have $\nu_{n} \rightarrow \mu$ weakly. For the details we refer to [19]. Conversely, let $\left(\nu_{n}\right)_{n \in \mathbb{N}}$ be a discrete skew convolution semigroup with infinitely divisible $\nu=\nu_{1} \in \mathcal{M}^{1}\left(\mathbb{R}^{d}\right)$ and assume that $\nu_{n} \rightarrow \mu$ weakly (equivalently, $\nu$ possesses a finite logarithmic moment). Then $\mu$ is operator-semi-selfdecomposable and there exists a continuous semistable hemigroup $\left(\mu_{s, t}\right)_{0 \leq s \leq t}$ with $\mu_{0,1}=\mu$ and $\nu_{n}=\mu_{c^{-n}, 1}$. The following construction is due to $[1,19]$. For $s>0$ let $n_{s}=\left\lfloor\log _{c} s\right\rfloor \in \mathbb{Z}$ and $r_{s}=s / c^{n_{s}} \in[1, c)$. With $\nu_{0}=\varepsilon_{0}$ we define

$$
\mu_{s, t}= \begin{cases}c^{\left(n_{s}+1\right) Q} \nu^{\log _{c} \frac{c}{r_{s}}} * c^{n_{t} Q} \nu_{n_{t}-n_{s}-1} * c^{\left(n_{t}+1\right) Q} \nu^{\log _{c} r_{t}} & \text { if } n_{t}>n_{s} \\ c^{\left(n_{t}+1\right) Q} \nu^{\log _{c} \frac{r_{t}}{r_{s}}} & \text { if } n_{t}=n_{s} \\ c^{n_{t} Q} \mu * c^{\left(n_{t}+1\right) Q} \nu^{\log _{c} r_{t}} & \text { if } s=0\end{cases}
$$

It is a straightforward calculation that $\left(\mu_{s, t}\right)_{0 \leq s \leq t}$ is indeed a continuous semistable hemigroup with the desired properties. Note that for the above construction the assumption that $\nu$ is infinitely divisible is essential. In contrast to stable hemigroups, Theorem 1.1 in [21] shows the existence of an infinitely divisible semi-selfdecomposable $\mu$ with cofactor $\nu=\nu_{1}$ not being infinitely divisible. Hence infinite divisibility of $\nu$ is a sufficient but not necessary condition for embeddability into a continuous semistable hemigroup.

Instead of using the embedding hemigroup (1.2) known from [1], it is advantageous to use an additive rather than a multiplicative parametrization. We will use the (additive) semistable hemigroup $\left(\lambda_{s, t}=\mu_{c^{-t}, c^{-s}}\right)_{0 \leq s \leq t}$ with $\nu_{n}=\mu_{c^{-n}, 1}=\lambda_{0, n} \rightarrow \mu$ weakly. One can easily show that (1.2) carries over to the simpler form

$$
\lambda_{s, t}= \begin{cases}c^{-\lfloor s\rfloor Q} \nu^{t-s} & \text { if }\lfloor s\rfloor=\lfloor t\rfloor \\ c^{-\lfloor t\rfloor Q} \lambda_{0, t-\lfloor t\rfloor} * c^{-Q} \nu_{\lfloor t\rfloor-1} * \nu^{1-s} & \text { if } 0 \leq s \leq 1 \leq t \\ c^{-\lfloor s\rfloor Q} \lambda_{s-\lfloor s\rfloor, t-\lfloor s\rfloor} & \text { else }\end{cases}
$$

In the following we extend and generalize these results to locally compact groups $\mathbb{G}$. In fact, for investigations of (semi-)selfdecomposability the assumption that the norming operators act contracting is essential. Furthermore the existence of continuous one-parameter groups of automorphisms implies connectedness. Therefore, without loss of generality, we assume $\mathbb{G}$ to be connected and contractible, hence a 
homogeneous group; cf. [9], 3.1.5 and Theorem 2.1.12. We first focus on the question of embeddability of a discrete skew convolution semigroup into a Lipschitz continuous semistable hemigroup in Section 2. As in the case of a stable hemigroup, the space-time enlargement enables us in Section 3 to obtain a Mehler hemigroup as a weak representation of the periodically stationary Ornstein-Uhlenbeck type process, and further a periodic hemigroup as a weak representation of the background driving additive periodic process. Finally, we show in Section 4 how to obtain weak analogues of random integral representations on $\mathbb{R}^{d}$ by generalized Lie-Trotter formulas for convolutions on $\mathbb{G}$.

\section{Hemigroup EMBEDding}

A close look at the embedding semistable hemigroup (1.3) on $\mathbb{R}^{d}$ shows that due to infinite divisibility of $\nu=\nu_{1}$ we fill the gaps left by the discrete skew convolution semigroup $\left(\nu_{n}\right)_{n \in \mathbb{N}}$ with the help of the semigroup $\left(\nu^{t}\right)_{t \geq 0}$. On non-Abelian groups $\mathbb{G}$ the assumption that $\nu$ is embeddable into a convolution semigroup is too restrictive and we rather prefer a more general hemigroup embedding. Recall that now we use additive parametrization and thus on $\mathbb{G}$ the objects under use have slightly different definitions below than given in the Introduction. In the following let $\mathbb{G}$ denote a homogeneous group, i.e. a contractible simply connected nilpotent Lie group. Let throughout $\tau \in \operatorname{Aut}(\mathbb{G})$ and let $\left(T_{t}\right)_{t \in \mathbb{R}}$ be a continuous one-parameter group in $\operatorname{Aut}(\mathbb{G})$.

Definition 2.1. (a) A family $\left(\nu_{s, t}\right)_{0 \leq s \leq t} \subseteq \mathcal{M}^{1}(\mathbb{G})$ is called a continuous hemigroup if $(s, t) \mapsto \nu_{s, t}$ is weakly continuous, $\nu_{s, s}=\varepsilon_{e}$ for all $s \geq 0$ and we have $\nu_{s, r} * \nu_{r, t}=\nu_{s, t}$ for all $0 \leq s \leq r \leq t$. The hemigroup is called $\tau$-semistable if $\tau\left(\nu_{s, t}\right)=\nu_{s+1, t+1}$ for all $0 \leq s \leq t$.

(b) A discrete hemigroup is a family $\{\nu(k, n)\}_{0 \leq k \leq n} \subseteq \mathcal{M}^{1}(\mathbb{G})$ with $k, n \in \mathbb{Z}_{+}$satisfying $\nu(k, k)=\varepsilon_{e}$ and $\nu(k, m) * \nu(m, n)=\nu(k, n)$ for all $0 \leq k \leq m \leq n$. Obviously, in this case $\nu(k, n)=*_{j=k+1}^{n} \nu(j-1, j)$, hence any sequence $\left\{\nu_{j}=\nu(j-1, j)\right\}_{j \in \mathbb{Z}_{+}}$ generates a discrete hemigroup. The discrete hemigroup is called $\tau$-semistable if $\tau(\nu(k, n))=\nu(k+1, n+1)$ for all $0 \leq k \leq n$.

Definition 2.2. (a) A weakly continuous family $\left(\nu_{t}\right)_{t \in \mathbb{R}_{+}} \subseteq \mathcal{M}^{1}(\mathbb{G})$ is called a skew convolution semigroup with respect to $\left(T_{t}\right)_{t \in \mathbb{R}}$ (or M-semigroup in Hazod [8]) if $\nu_{s+t}=\nu_{s} * T_{s}\left(\nu_{t}\right)$ for all $s, t \geq 0$.

(b) A sequence $\{\nu(k)\}_{k \in \mathbb{Z}_{+}} \subseteq \mathcal{M}^{1}(\mathbb{G})$ is called a discrete skew convolution semigroup with respect to $\tau \in \operatorname{Aut}(\mathbb{G})$ if $\nu(0)=\varepsilon_{e}$ and $\nu(k+n)=\nu(k) * \tau^{k}(\nu(n))$ for all $k, n \in \mathbb{Z}_{+}$.

As in the continuous case, discrete semistable hemigroups and discrete skew convolution semigroups are closely related. One immediately verifies the following relations.

Proposition 2.3. (a) $\{\nu(k)\}_{k \in \mathbb{Z}_{+}}$is a discrete skew convolution semigroup iff $\nu(0)=$ $\varepsilon_{e}$ and $\nu(k)=*_{j=1}^{k} \tau^{j-1}(\nu)$ for all $k \in \mathbb{N}$ with $\nu=\nu(1)$. 
(b) $\{\nu(k, n)\}_{0 \leq k \leq n}$ is a discrete $\tau$-semistable hemigroup iff $\nu(k, k)=\varepsilon_{e}$ and $\nu(k, n)=$ $*_{j=k+1}^{n} \tau^{j-1}(\nu)$ for all $0 \leq k<n$ with $\nu=\nu(0,1)$.

(c) $\{\nu(k)\}_{k \in \mathbb{Z}_{+}}$is a discrete skew convolution semigroup with respect to $\tau$ iff $\{\nu(k, n)=$ $\left.\tau^{k}(\nu(n-k))\right\}_{0 \leq k \leq n}$ is a discrete $\tau$-semistable hemigroup.

Remark 2.4. According to Proposition 2.3 any $\nu \in \mathcal{M}^{1}(\mathbb{G})$ may be embedded into a discrete skew convolution semigroup, respectively a discrete $\tau$-semistable hemigroup. Therefore we first concentrate on the problem under which conditions a discrete $\tau$ semistable hemigroup may be embedded into a continuous $\tau$-semistable hemigroup. We call $\nu \in \mathcal{M}^{1}(\mathbb{G})$ embeddable into a hemigroup (for short: h-embeddable) if there exists a continuous hemigroup $\left(\mu_{s, t}\right)_{0 \leq s \leq t \leq 1}$ with $\mu_{0,1}=\nu$.

Note that h-embeddable laws $\nu=\mu_{0,1}$ with a commuting hemigroup, i.e. $\mu_{s, t} * \mu_{u, v}=$ $\mu_{u, v} * \mu_{s, t}$ for $(s, t] \cap(u, v]=\emptyset$, are infinitely divisible as limits of commuting infinitesimal triangular arrays, and hence embeddable into a continuous convolution semigroup; cf. Shah [23], Theorem 1.1.

A hemigroup $\left(\nu_{s, t}\right)_{0 \leq s \leq t}$ is called 1-periodic if $\nu_{s+1, t+1}=\nu_{s, t}$ for all $0 \leq s \leq t$. Obviously, any h-embeddable law $\nu$ is embeddable into a 1-periodic hemigroup. If $\nu$ is embeddable into a continuous convolution semigroup $\left(\rho_{t}\right)_{t \geq 0}$ with $\rho_{1}=\nu$ then $\left(\nu_{s, t}=\rho_{t-s}\right)_{0 \leq s \leq t}$ is obviously 1-periodic.

Proposition 2.5. (a) Embedding of a discrete hemigroup $\{\nu(k, n)\}_{0 \leq k \leq n}$ into a continuous hemigroup is possible iff all $\nu_{j}=\nu(j-1, j)$ are $h$-embeddable.

(b) Embedding of a discrete semistable hemigroup $\{\nu(k, n)\}_{0 \leq k \leq n}$ into a continuous semistable hemigroup is possible iff $\nu=\nu(0,1)$ is h-embeddable.

Proof. The proof of (a) is obvious. To prove (b) first observe that if $\left(\lambda_{s, t}\right)_{0 \leq s \leq t}$ is a semistable hemigroup with $\lambda_{k, n}=\nu(k, n)$ for all $k, n \in \mathbb{Z}_{+}, k \leq n$, then $\left(\mu_{s, t}=\right.$ $\left.\lambda_{s, t}\right)_{0 \leq s \leq t \leq 1}$ is a continuous hemigroup with $\mu_{0,1}=\lambda_{0,1}=\nu(0,1)=\nu$. Conversely, let $\nu$ be h-embeddable with $\nu=\mu_{0,1}$ and $\nu(k, n)=*_{j=k+1}^{n} \tau^{j-1}(\nu)$ for all $k, n \in \mathbb{Z}_{+}$, $k<n$. Define

$$
\lambda_{s, t}= \begin{cases}\tau^{\lfloor s\rfloor}\left(\mu_{s-\lfloor s\rfloor, t-\lfloor s\rfloor}\right) & , \text { if }\lfloor s\rfloor=\lfloor t\rfloor \\ \mu_{s, 1} * \nu(1,\lfloor t\rfloor) * \tau^{\lfloor t\rfloor}\left(\mu_{0, t-\lfloor t\rfloor}\right) & , \text { if } 0 \leq s \leq 1 \leq t \\ \tau^{\lfloor s\rfloor}\left(\lambda_{s-\lfloor s\rfloor, t-\lfloor s\rfloor}\right) & , \text { else. }\end{cases}
$$

As easily verified, $\left(\lambda_{s, t}\right)_{0 \leq s \leq t}$ is a continuous hemigroup with $\lambda_{k, n}=\nu(k, n)$ for all $k, n \in \mathbb{Z}_{+}, k \leq n$, and we have $\tau\left(\lambda_{s, t}\right)=\lambda_{s+1, t+1}$ by construction.

Note that (2.1) coincides with the semistable hemigroup (1.3) on $\mathbb{G}=\mathbb{R}^{d}$ if we set $\tau=c^{-Q}$ and $\mu_{s, t}=\nu^{t-s}$ in case $\nu$ is infinitely divisible.

Now, according to Siebert [24], let $\left(X_{i}\right)_{i=1}^{d}$ be a basis of the Lie algebra $\mathfrak{G}$ of $\mathbb{G}$, and let $\left(\xi_{i}\right)_{i=1}^{d}$ be a local coordinate system, i.e. $\xi_{i} \in \mathcal{C}_{c}^{\infty}(\mathbb{G})$ with $\left|\xi_{i}\right| \leq 1, \xi_{i}(e)=0$, $\xi_{i}\left(x^{-1}\right)=-\xi_{i}(x)$ and $X_{i}\left(\xi_{j}\right)=\delta_{i j}$. Furthermore choose a Hunt function $\varphi=\varphi_{\mathbb{G}}$ with $\varphi=\sum_{i=1}^{d} \xi_{i}^{2}$ on a compact neighbourhood $U_{0}$ of $e$ such that $0 \leq \varphi \leq 1$ and 
$1-\varphi \in \mathcal{C}_{c}^{\infty}(\mathbb{G})$. Let $\mathbb{A}(\mathbb{G})$ denote the cone of generating functionals of continuous convolution semigroups. For the background of probabilities on groups and LévyKhintchine formulas see e.g. [11, 9].

Definition 2.6. (a) For $\mu \in \mathcal{M}^{1}(\mathbb{G})$ we define $q(\mu)=\sum_{i=1}^{d}\left|\left\langle\mu, \xi_{i}\right\rangle\right|+\langle\mu, \varphi\rangle$ called the $q$-functional. Similarly, for a generating functional $A \in \mathbb{A}(\mathbb{G})$ we define $\|A\|=\sum_{i=1}^{d}\left|\left\langle A, \xi_{i}\right\rangle\right|+\langle A, \varphi\rangle$ and hence for the Poisson generator $A=\mu-\varepsilon_{e}$ we may write $q(\mu)=\|A\|=\left\|\mu-\varepsilon_{e}\right\|$.

(b) A hemigroup $\left(\mu_{s, t}\right)_{0 \leq s \leq t}$ is called Lipschitz continuous on $[0, R]$ if $q\left(\mu_{s, t}\right) \leq$ $C(t-s)$ for all $0 \leq s \leq t \leq R$, where $C>0$ depends on the hemigroup and on $R$. We simply call the hemigroup Lipschitz continuous if this condition is fulfilled for every $R>0$.

(c) A hemigroup $\left(\mu_{s, t}\right)_{0 \leq s \leq t}$ is called of bounded variation on $[0, R]$ if for all decompositions $0=r_{1}<r_{2}<\cdots<r_{n}<r_{n+1}=R$ we have $\sum_{i=1}^{n} q\left(\mu_{r_{i}, r_{i+1}}\right) \leq \gamma$, where $\gamma>0$ depends on the hemigroup and on $R$. We simply call the hemigroup of bounded variation if this condition is fulfilled for every $R>0$.

Remark 2.7. (a) As mentioned in 2.5 of Siebert [24], $\|A\|$ is equivalent to $|A|_{2}$ and $|A|_{2}^{\sim}$, the norms of the functional $A$ on the spaces of twice differentiable functions $\mathcal{C}_{2}(\mathbb{G})$, respectively $\mathcal{C}_{2}^{\sim}(\mathbb{G})$. Therefore it easily follows that for $T \in \operatorname{Aut}(\mathbb{G})$ there exists $C(T)>0$ such that $q(T(\mu))=\left\|T\left(\mu-\varepsilon_{e}\right)\right\| \leq C(T) q(\mu)$ for $\mu \in \mathcal{M}^{1}(\mathbb{G})$ and $\|T(A)\| \leq C(T)\|A\|$ for $A \in \mathbb{A}(\mathbb{G})$. Hence, according to Proposition 2.5, the semistable hemigroup $\left(\lambda_{s, t}\right)_{0 \leq s \leq t}$ is Lipschitz continuous on any interval $[0, R]$ iff $\left(\mu_{s, t}=\lambda_{s, t}\right)_{0 \leq s \leq t \leq 1}$ is Lipschitz continuous.

(b) Lipschitz continuous hemigroups are almost surely differentiable by Siebert [24], Theorem 4.3 and Lemma 2.8. This fact will be important in Section 3 for the construction of the background driving process. For continuously differentiable hemigroups a different approach is given by Kunita [16].

Theorem 2.8. Let $\nu \in \mathcal{M}^{1}(\mathbb{G})$ be h-embeddable with $\nu=\mu_{0,1}$ for some continuous hemigroup $\left(\mu_{s, t}\right)_{0 \leq s \leq t \leq 1}$. Then there exists a Lipschitz continuous hemigroup $\left(\mu_{s, t}^{\prime}\right)_{0 \leq s \leq t \leq 1}$ with $\mu_{0,1}^{\prime}=\nu=\mu_{0,1}$.

Proof. Step 1: We first show that there exists a continuous hemigroup of bounded variation $\left(\bar{\mu}_{s, t}\right)_{0 \leq s \leq t \leq 1}$ with $\bar{\mu}_{0,1}=\nu$.

The continuous hemigroup $\left(\mu_{s, t}\right)_{0 \leq s \leq t \leq 1}$ may be represented as the family of distributions of the increments $X_{s}^{-1} \bar{X}_{t}$ of a stochastically continuous additive process $\left(X_{t}\right)_{t \in[0,1]}$ with $X_{0}=e$ almost surely. According to Feinsilver [6], Section 3e), there exists a decomposition $X_{t}=Z_{t} \cdot m_{t}$, where $t \mapsto m_{t} \in \mathbb{G}$ is continuous and $\left(Z_{t}\right)_{t \in[0,1]}$ is an additive process with $Z_{0}=e$ almost surely such that the Lévy-Khintchine characteristics of the corresponding generating functionals are of bounded variation. In fact, $\left(Z_{t}\right)_{t \in[0,1]}$ is characterized by the property that $t \mapsto f\left(Z_{t}\right)-\int_{0}^{t} f\left(Z_{s}\right) L(d s)$ is a martingale for every $f \in \mathcal{C}_{c}^{\infty}(\mathbb{G})$, where $L$ is given by the covariance function and the Lévy-measure function. For details cf. Feinsilver [6]. Let $\left(\nu_{s, t}\right)_{0 \leq s \leq t \leq 1}$ denote 
the family of distributions of the increments of $\left(Z_{t}\right)_{t \in[0,1]}$. Since the generating functionals of $\left(\nu_{s, t}\right)_{0 \leq s \leq t \leq 1}$ are of bounded variation, the hemigroup itself is of bounded variation in the sense of Definiton 2.6(c), cf. Heyer and Pap[12], Theorems 6 and 7. For $0 \leq s \leq t \leq 1$ we have $X_{s}^{-1} X_{t}=m_{s}^{-1} Z_{s}^{-1} Z_{t} \cdot m_{t}$ and hence $\mu_{s, t}=\varepsilon_{m_{s}^{-1}} * \nu_{s, t} * \varepsilon_{m_{t}}$, especially $\mu_{0,1}=\nu_{0,1} * \varepsilon_{m_{1}}$, since $m_{0}=e$. Now choose a continuous one-parameter group $(u(t))_{t \in \mathbb{R}} \subseteq \mathbb{G}$ with $u(1)=m_{1}$ and define

$$
\bar{\mu}_{s, t}= \begin{cases}\nu_{2 s, 2 t} & , \text { if } 0 \leq s \leq t \leq \frac{1}{2} \\ \varepsilon_{u(2(t-s))} & , \text { if } \frac{1}{2} \leq s \leq t \leq 1 \\ \bar{\mu}_{s, \frac{1}{2}} * \bar{\mu}_{\frac{1}{2}, t} & , \text { if } 0 \leq s \leq \frac{1}{2} \leq t \leq 1\end{cases}
$$

Obviously, by construction $\left(\bar{\mu}_{s, t}\right)_{0 \leq s \leq t \leq 1}$ is a continuous hemigroup of bounded variation with $\bar{\mu}_{0,1}=\nu_{0,1} * \varepsilon_{m_{1}}=\mu_{0,1}=\nu$.

Step 2: There exists a Lipschitz continuous hemigroup $\left(\mu_{s, t}^{\prime}\right)_{0 \leq s \leq t \leq 1}$ with $\mu_{0,1}^{\prime}=\bar{\mu}_{0,1}=$ $\nu$. This follows from Siebert [24], 7.4, with $\mu_{s, t}^{\prime}=\bar{\mu}_{U^{-1}(s), U^{-1}(t)}$ for a suitable function $U:[0,1] \rightarrow[0,1]$.

We now turn to the behaviour at infinity which is closely related to semiselfdecomposability. Essentially, in the following we will need $\tau$ to be contracting, i.e. $\tau^{n}(x) \rightarrow e$ for all $x \in \mathbb{G}$.

Definition 2.9. A probability measure $\mu \in \mathcal{M}^{1}(\mathbb{G})$ is called $\tau$-semiselfdecomposable if there exists a $\tau$-semistable hemigroup $\left(\lambda_{s, t}\right)_{0 \leq s \leq t}$ such that $\lambda_{0, t} \rightarrow \mu$ weakly as $t \rightarrow \infty$.

Obviously, for $n \in \mathbb{N}$ and $t>n$ we get

$$
\lambda_{0, t}=\lambda_{0, n} * \lambda_{n, t}=\nu(n) * \tau^{n}\left(\lambda_{0, t-n}\right) \rightarrow \nu(n) * \tau^{n}(\mu)=\mu
$$

weakly as $t \rightarrow \infty$, coinciding with the usual definition of semi-selfdecomposability. Since $\lambda_{\lfloor t\rfloor, t}=\tau^{\lfloor t\rfloor}\left(\lambda_{0, t-\lfloor t\rfloor}\right) \rightarrow \varepsilon_{e}$ by contractivity, due to the above hemigroup embedding we obtain that $\mu$ is $\tau$-semi-selfdecomposable iff there exists an h-embeddable law $\nu$ such that $\nu(n)=*_{k=0}^{n-1} \tau^{k}(\nu) \rightarrow \mu$ weakly. According to [10], $\nu(n)$ converges weakly iff $\nu$ possesses a finite logarithmic moment, i.e. $\int_{\mathbb{G}} \log (1+\|x\|) d \nu(x)<\infty$, where $\|\cdot\|$ is a norm on the homogeneous group. On $\mathbb{G}=\mathbb{R}^{d}$ this coincides with the assumption of a finite logarithmic moment in $[2,20]$.

\section{Space-time enlargement and Mehler hemigroups}

According to Proposition 2.5(b) and Theorem 2.8, in the sequel we assume without loss of generality that for h-embeddable laws the underlying semistable hemigroup is Lipschitz continuous, in particular the semistable hemigroup constructed in (2.1). Moreover, for $\tau \in \operatorname{Aut}(\mathbb{G})$ there exists a continuous one-parameter group $\left(T_{t}\right)_{t \in \mathbb{R}} \subseteq \operatorname{Aut}(\mathbb{G})$ such that $\tau^{k}=T_{1}$ for some $k \in \mathbb{N}$; cf. [9], Proposition 2.8.14. If $\tau$ is contracting then also $\left(T_{t}\right)_{t \in \mathbb{R}}$ is contracting. Hence in the sequel we further assume 
without loss of generality $\tau$ to be embedded into a continuous one-parameter group $\left(T_{t}\right)_{t \in \mathbb{R}}$ with $\tau=T_{1}$.

Following an idea of Hofmann and Jurek [13], let $\mathbb{H}=\mathbb{G} \rtimes \mathbb{R}$ denote the space-time group, where the semidirect product is defined by the action $\left(T_{t}\right)_{t \in \mathbb{R}}$ as

$$
(x, s) \cdot(y, t)=\left(x \cdot T_{s}(y), s+t\right) \quad \text { for all } x, y \in \mathbb{G} \text { and } s, t \in \mathbb{R},
$$

cf.,e.g., [9], §2.14 III. Let $\mathcal{M}_{\star}^{1}(\mathbb{H})=\left\{\mu \otimes \varepsilon_{u}: \mu \in \mathcal{M}^{1}(\mathbb{G}), u \in \mathbb{R}\right\}$ be the closed, convex subsemigroup of $\mathcal{M}^{1}(\mathbb{H})$ with convolution given by

$$
\left(\mu \otimes \varepsilon_{s}\right) \star\left(\nu \otimes \varepsilon_{t}\right)=\left(\mu * T_{s}(\nu), \varepsilon_{s+t}\right) \quad \text { for } \mu, \nu \in \mathcal{M}^{1}(\mathbb{G}) \text { and } s, t \in \mathbb{R} .
$$

Let $\left(\lambda_{s, t}\right)_{0 \leq s \leq t} \subseteq \mathcal{M}^{1}(\mathbb{G})$ be a continuous hemigroup and define

$$
\begin{aligned}
\lambda_{s, t}^{\bullet} & =T_{-s}\left(\lambda_{s, t}\right) \subseteq \mathcal{M}^{1}(\mathbb{G}) \\
\Lambda_{s, t} & =\lambda_{s, t}^{\bullet} \otimes \varepsilon_{t-s} \subseteq \mathcal{M}_{\star}^{1}(\mathbb{H})
\end{aligned}
$$

then one can easily verify that $\left(\Lambda_{s, t}\right)_{0 \leq s \leq t}$ is a continuous hemigroup. Conversely, let $\left(\Lambda_{s, t}^{\prime}=\lambda_{s, t}^{\prime} \otimes \varepsilon_{\varphi(s, t)}\right)_{0 \leq s \leq t} \subseteq \mathcal{M}_{\star}^{1}(\mathbb{H})$ be a continuous hemigroup. Then for $0 \leq s \leq r \leq$ $t$ we have $\varphi(s, r)+\varphi(r, t)=\varphi(s, t)$ and hence, with $\psi(t)=\varphi(0, t)$ we get $\varphi(s, t)=$ $\psi(t)-\psi(s)$. Furthermore $\lambda_{s, r}^{\prime} * T_{\psi(r)-\psi(s)}\left(\lambda_{r, t}^{\prime}\right)=\lambda_{s, t}^{\prime}$ and thus $\left(\lambda_{s, t}^{\prime \prime}=T_{\psi(s)}\left(\lambda_{s, t}^{\prime}\right)\right)_{0 \leq s \leq t}$ is a continuous hemigroup in $\mathcal{M}^{1}(\mathbb{G})$.

For bounded measurable functions $g: \mathbb{H} \rightarrow \mathbb{R}$ we obtain the convolutions

$$
\begin{aligned}
\Lambda_{s, t} \star g(x, r) & =\int_{\mathbb{H}} g((y, u) \cdot(x, r)) d \Lambda_{s, t}(y, u) \\
& =\int_{\mathbb{G}} g\left(y \cdot T_{t-s}(x), t-s+r\right) d \lambda_{s, t}^{\bullet}(y) \\
& =\int_{\mathbb{G}} g\left(T_{-s}\left(y \cdot T_{t}(x)\right), t-s+r\right) d \lambda_{s, t}(y) .
\end{aligned}
$$

Thus for the space component we may define a family $\left(P_{s, t}\right)_{0 \leq s \leq t}$ of bounded linear operators as

$$
P_{s, t}(f)(x)=\int_{\mathbb{G}} f\left(T_{-s}\left(y \cdot T_{t}(x)\right)\right) d \lambda_{s, t}(y)
$$

for bounded measurable functions $f: \mathbb{G} \rightarrow \mathbb{R}$. As easily verified we have the following properties.

Lemma 3.1. For a $\tau$-semistable hemigroup $\left(\lambda_{s, t}\right)_{0 \leq s \leq t}$ the above family $\left(P_{s, t}\right)_{0 \leq s \leq t}$ is a 1-periodic Feller hemigroup, i.e.

$$
\begin{aligned}
& P_{r, t} P_{s, r}=P_{s, t} \quad \text { for all } 0 \leq s \leq r \leq t . \\
& P_{s+1, t+1}=P_{s, t} \quad \text { for all } 0 \leq s \leq t . \\
& P_{s, t}(f) \in \mathcal{C}_{b}(\mathbb{G}) \quad \text { for every } f \in \mathcal{C}_{b}(\mathbb{G}) \text { and } 0 \leq s \leq t .
\end{aligned}
$$


In analogy to Mehler semigroups for stable hemigroups, we call $\left(P_{s, t}\right)_{0 \leq s \leq t}$ a Mehler hemigroup of linear operators. These operators are closely related to the background driving process, which will be of our interest in the sequel.

Proposition 3.2. (a) The hemigroup $\left(\lambda_{s, t}\right)_{0 \leq s \leq t} \subseteq \mathcal{M}^{1}(\mathbb{G})$ is Lipschitz continuous iff $\left(\Lambda_{s, t}\right)_{0 \leq s \leq t} \subseteq \mathcal{M}_{\star}^{1}(\mathbb{H})$ shares this property. This is the case iff the function $(s, t) \mapsto$ $\lambda_{s, t}^{\bullet} \in \mathcal{M}^{\overline{1}}(\bar{G})$ is Lipschitz continuous.

(b) The hemigroup $\left(\lambda_{s, t}\right)_{0 \leq s \leq t}$ is $\tau$-semistable iff $\left(\Lambda_{s, t}\right)_{0 \leq s \leq t}$ is 1-periodic. This is the case iff the function $(s, t) \mapsto \lambda_{s, t}^{\bullet} \in \mathcal{M}^{1}(\mathbb{G})$ is 1-periodic.

Proof. As in Definition 2.6, let $\left(\xi_{i}\right)_{i=1}^{d}$ be a local coordinate system on $\mathbb{G}$ and let $\varphi_{\mathbb{G}}$ be a Hunt function. Further let $\xi_{d+1}$ be a local coordinate function on $\mathbb{R}$, e.g. $\xi_{d+1}(t)=$ $\frac{t}{1+t^{2}}$. Then $\left(\bar{\xi}_{i}\right)_{i=1}^{d+1}$ with $\bar{\xi}_{i}(x, t)=\xi_{i}(x)$ for $1 \leq i \leq d$ and $\bar{\xi}_{d+1}(x, t)=\xi_{d+1}(t)$ defines a local coordinate system on $\mathbb{H}$ and $\varphi_{\mathbb{H}}(x, t)=\varphi_{\mathbb{G}}(x)+\xi_{d+1}^{2}(t)$ is a Hunt function on $\mathbb{H}$. The $q$-functional on the space-time group is then given by $q_{\mathbb{H}}\left(\mu \otimes \varepsilon_{t}\right)=$ $q_{\mathbb{G}}(\mu)+\left|\xi_{d+1}(t)\right|+\xi_{d+1}^{2}(t)$. Together with Remark 2.7 this proves (a), and (b) is easily verified by direct calculation.

According to Siebert [24], Theorem 4.3 and Lemma 2.8, the Lipschitz continuous semistable hemigroup $\left(\lambda_{s, t}\right)_{0 \leq s \leq t}$ constructed in (2.1) is almost surely differentiable. For $0 \leq s \leq 1$ let $C(s)=\left.\frac{\partial^{+}}{\partial t} \mu_{s, t}\right|_{t=s} \in \mathbb{A}(\mathbb{G})$. Then, by the construction in (2.1), for $s>0$ we obtain

$$
A(s)=\left.\frac{\partial^{+}}{\partial t} \lambda_{s, t}\right|_{t=s}=T_{\lfloor s\rfloor}\left(\left.\frac{\partial^{+}}{\partial t} \mu_{s-\lfloor s\rfloor, t-\lfloor s\rfloor}\right|_{t=s}\right)=T_{\lfloor s\rfloor}(C(s-\lfloor s\rfloor)) .
$$

The almost everywhere defined mapping $s \mapsto A(s) \in \mathbb{A}(\mathbb{G})$ is admissible in the sense of Siebert [24], 2.6, and we have

$$
B(s, t)=B(t)-B(s)=\int_{s}^{t} A(u) d u=\int_{s}^{t} T_{\lfloor u\rfloor}(C(u-\lfloor u\rfloor)) d u \in \mathbb{A}(\mathbb{G}),
$$

where $t \mapsto B(t)=B(0, t)$ is increasing and Lipschitz continuous. On the other hand, again by Siebert [24], Theorem 4.3 and Lemma 2.8, and by Proposition 3.2(a), $\left(\Lambda_{s, t}\right)_{0 \leq s \leq t}$ is almost surely differentiable, hence in particular, $\left(\lambda_{s, t}^{\bullet}\right)_{0 \leq s \leq t}$ is almost surely differentiable. Put $\bar{A}(s)=\left.\frac{\partial^{+}}{\partial t} \Lambda_{s, t}\right|_{t=s} \in \mathbb{A}(\mathbb{H})$ then for the space component we obtain

$$
A^{\bullet}(s)=\left.\frac{\partial^{+}}{\partial t} \lambda_{s, t}^{\bullet}\right|_{t=s}=T_{-s} A(s)=T_{-(s-\lfloor s\rfloor)}(C(s-\lfloor s\rfloor)) .
$$

As above, we further define the generating functionals

$$
\begin{array}{r}
\bar{B}(s, t)=\bar{B}(t)-\bar{B}(s)=\int_{s}^{t} \bar{A}(u) d u \in \mathbb{A}(\mathbb{H}) \quad \text { with } \bar{B}(t)=\bar{B}(0, t) \\
B^{\bullet}(s, t)=B^{\bullet}(t)-B^{\bullet}(s)=\int_{s}^{t} A^{\bullet}(u) d u \in \mathbb{A}(\mathbb{G}) \quad \text { with } B^{\bullet}(t)=B^{\bullet}(0, t)
\end{array}
$$

and we easily obtain the following relations. 
Proposition 3.3. $\tau$-semistability, respectively 1-periodicity of the hemigroups imply for all $0 \leq s \leq t$

$$
\begin{array}{rll}
A(s+1)=\tau(A(s)) & \text { and } & B(s+1, t+1)=\tau(B(s, t)), \\
\bar{A}(s+1)=\bar{A}(s) \quad \text { and } & \bar{B}(s+1, t+1)=\bar{B}(s, t), \\
A^{\bullet}(s+1)=A^{\bullet}(s) \quad & \text { and } & B^{\bullet}(s+1, t+1)=B^{\bullet}(s, t) .
\end{array}
$$

Now we are ready to prove

Theorem 3.4. There exists a bijection between Lipschitz continuous $\tau$-semistable hemigroups $\left(\lambda_{s, t}\right)_{0 \leq s \leq t}$ and Lipschitz continuous 1-periodic hemigroups $\left(\bar{\lambda}_{s, t}\right)_{0 \leq s \leq t}$ on $\mathcal{M}^{1}(\mathbb{G})$ given by their families of generating functionals $(B(s, t))_{0 \leq s \leq t}$, repectively $\left(B^{\bullet}(s, t)\right)_{0 \leq s \leq t}$.

Remark 3.5. In analogy to background driving Lévy processes for stable hemigroups, we call $\left(\bar{\lambda}_{s, t}\right)_{0 \leq s \leq t}$ the (family of distributions of the increments of the) background driving additive periodic process.

Proof. According to Proposition 3.2 we have a 1-1-correspondence between Lipschitz continuous $\tau$-semistable hemigroups $\left(\lambda_{s, t}\right)_{0<s<t} \subseteq \mathcal{M}^{1}(\mathbb{G})$ and Lipschitz continuous 1-periodic hemigroups $\left(\Lambda_{s, t}\right)_{0 \leq s \leq t} \subseteq \mathcal{M}^{1}(\mathbb{H})$. According to Siebert [24], Section 4, these hemigroups are uniquely determined by the families of generating functionals $(B(s, t))_{0 \leq s \leq t}$, repectively $(\bar{B}(s, t))_{0 \leq s \leq t}$, or the corresponding admissible families $(A(u))_{u \geq 0} \subseteq \mathbb{A}(\mathbb{G})$, respectively $(\bar{A}(u))_{u \geq 0} \subseteq \mathbb{A}(\mathbb{H})$, satisfying the evolution equations (EE2) (Siebert [24], 4.3), respectively condition (E) (Siebert [24], 3.6). As easily seen, since $\Lambda_{s, t} \in \mathcal{M}_{\star}^{1}(\mathbb{H}),(\bar{B}(t))_{t \geq 0}$ satisfies (E), respectively $(\bar{A}(u))_{u \geq 0}$ satisfies (EE2) iff $\left(B^{\bullet}(t)\right)_{t \geq 0}$, respectively $\left(A^{\bullet}(u)\right)_{u \geq 0}$ satisfy these conditions. Therefore, by Siebert [24], 5.7 or 5.10 , there exists a uniquely determined Lipschitz continuous hemigroup $\left(\bar{\lambda}_{s, t}\right)_{0 \leq s \leq t}$ with generating functionals $\left(B^{\bullet}(s, t)=B^{\bullet}(t)-B^{\bullet}(s)\right)_{0 \leq s \leq t}$, i.e. $A^{\bullet}(s)=$ $\left.\frac{\partial^{+}}{\partial t} \bar{\lambda}_{s, t}\right|_{t=s}$ almost everywhere. By Proposition 3.3 we conclude $A^{\bullet}(s+1)=A^{\bullet}(s)$ and $B^{\bullet}(s+1, t+1)=B^{\bullet}(s, t)$ for all $0 \leq s \leq t$. Furthermore (cf. Siebert [24], 6.1), for $R>0$ and a sequence of decompositions $0=c_{0}^{(n)}<c_{1}^{(n)}<\cdots<c_{n-1}^{(n)}<c_{n}^{(n)}=R$ with $\max _{1 \leq i \leq n}\left|c_{i}^{(n)}-c_{i-1}^{(n)}\right| \rightarrow 0$, we have

$$
\bar{\lambda}_{s, t}=\lim _{n \rightarrow \infty} \stackrel{r_{n}(t)}{\underset{i}{*} \boldsymbol{*}} \underset{r_{n}(s)+1}{\operatorname{Exp}}\left(B^{\bullet}\left(c_{i}^{(n)}, c_{i+1}^{(n)}\right)\right),
$$

where $(\operatorname{Exp}(t U))_{t \geq 0}$ denotes the convolution semigroup generated by $U \in \mathbb{A}(\mathbb{G})$ and $r_{n}(u)=k$ iff $c_{k}^{(n)} \leq u<c_{k+1}^{(n)}$. Therefore, 1-periodicity of $\left(B^{\bullet}(s, t)\right)_{0 \leq s \leq t}$ implies 1periodicity of $\left(\bar{\lambda}_{s, t}\right)_{0 \leq s \leq t}$ as asserted.

The converse is proved analogously. Given $A^{\bullet}(s)=\left.\frac{\partial^{+}}{\partial t} \bar{\lambda}_{s, t}\right|_{t=s}$ we define $A(s)=$ 
$T_{s}\left(A^{\bullet}(s)\right)$ and $B(s, t)=\int_{s}^{t} A(u) d u$. Then, observing (again by Siebert [24], 6.1)

$$
\lambda_{s, t}=\lim _{n \rightarrow \infty} \underset{i=r_{n}(s)+1}{\stackrel{r_{n}(t)}{*}} \operatorname{Exp}\left(B\left(c_{i}^{(n)}, c_{i+1}^{(n)}\right)\right)
$$

and noting that periodicity $B^{\bullet}(s+1, t+1)=B^{\bullet}(s, t)$ yields $B(s+1, t+1)=\tau(B(s, t))$, we conclude semistability $\tau\left(\lambda_{s, t}\right)=\lambda_{s+1, t+1}$.

\section{Representations By Generalized Lie-Trotter Formulas}

For vector spaces $\mathbb{G}=\mathbb{R}^{d}$ the additive periodic driving process can be represented by pathwise random integrals; cf. [2, 20]. For stable hemigroups on homogeneous groups the background driving process is a Lévy process and a weak version of random integrals is obtained by the Lie-Trotter formula for convolution semigroups, see [8, 9]. In order to obtain similar results for semistable hemigroups on homogeneous groups $\mathbb{G}$ we have to analyze Section 3 of Siebert [24]. There the hemigroups are represented as limits of row-products of infinitesimal arrays $\mu_{s, t}=\lim _{n \rightarrow \infty} *_{k=\lfloor n s\rfloor+1}^{\lfloor n t\rfloor} \sigma_{n, k}$. Crucial are the following conditions $\left(\mathrm{S}^{\prime}\right)$ and $(\mathrm{T})$ in Siebert [24]

$$
\sum_{k=1}^{n} q\left(\sigma_{n, k}\right) \leq \gamma \quad \text { for some } \gamma>0 \text { and all } n \in \mathbb{N} .
$$

For every $\varepsilon>0$ there exists a compact $K_{\varepsilon} \subseteq \mathbb{G}$ such that

$$
\sum_{k=1}^{n} \sigma_{n, k}\left(\complement K_{\varepsilon}\right)<\varepsilon \quad \text { for all } n \in \mathbb{N} .
$$

Here, in place of $\sigma_{n, k}$, for $k, n \in \mathbb{N}$ we consider the arrays given by

$$
\mu(n, k)=\lambda_{\frac{k-1}{n}, \frac{k}{n}} \quad \text { and } \quad \mu^{\bullet}(n, k)=\lambda_{\frac{k-1}{n}, \frac{k}{n}}^{\bullet}=T_{\frac{k-1}{n}}(\mu(n, k)),
$$

which obviously are infinitesimal.

Proposition 4.1. The arrays $\{\mu(n, k)\}_{k, n \in \mathbb{N}}$ and $\left\{\mu^{\bullet}(n, k)\right\}_{k, n \in \mathbb{N}}$ fulfill conditions (4.1) and (4.2) (conditions $\left(S^{\prime}\right)$ and $(T)$ in Siebert [24]).

Proof. Since $\left(\lambda_{s, t}\right)_{0 \leq s \leq t}$ is Lipschitz continuous, the array $\{\mu(n, k)\}_{k, n \in \mathbb{N}}$ satisfies condition (4.1); cf. Siebert [24], 5.3 and 5.4. Therefore, also $\left\{\mu^{\bullet}(n, k)\right\}_{k, n \in \mathbb{N}}$ satisfies condition (4.1) by Remark 2.7. To prove the tightness condition (4.2) we switch to the space-time hemigroup $\left(\Lambda_{s, t}\right)_{0 \leq s \leq t}$. Let $\varphi_{\mathbb{G}}$ and $\varphi_{\mathbb{H}}$ be Hunt functions as in the proof of Proposition 3.2, and let $\eta$ denote the Lévy-measure of $\bar{B}(0,1)=\bar{B}(1)$. Put

$$
\kappa(n, k)=\Lambda_{\frac{k-1}{n}, \frac{k}{n}}-\varepsilon_{(e, 0)} \quad \text { and } \quad \kappa_{n}(s, t)=\sum_{k=\lfloor n s\rfloor+1}^{\lfloor n t\rfloor} \kappa(n, k) .
$$


Then for all $0 \leq s \leq t$ we have $\kappa_{n}(s, t) \rightarrow \bar{B}(s, t)$ by Siebert [24], Theorem 3.6, hence for nonnegative $f \in \mathcal{C}_{2}(\mathbb{H})$ with $f(e, 0)=0$ we get

$$
\langle\bar{B}(1), f\rangle=\lim _{n \rightarrow \infty}\left\langle\sum_{k=1}^{n} \kappa(n, k), f\right\rangle=\lim _{n \rightarrow \infty}\left\langle\sum_{k=1}^{n} \mu^{\bullet}(n, k) \otimes \varepsilon_{n^{-1}}, f\right\rangle .
$$

Especially, for $f=\varphi_{\mathbb{H}}=\varphi_{\mathbb{G}}+\xi_{d+1}^{2}$ we have

$$
\left\langle\bar{B}(1), \varphi_{\mathbb{H}}\right\rangle=\lim _{n \rightarrow \infty}\left\langle\sum_{k=1}^{n} \mu^{\bullet}(n, k), \varphi_{\mathbb{G}}\right\rangle,
$$

since $\xi_{d+1}^{2}\left(n^{-1}\right) \rightarrow 0$. Furthermore, since $\Lambda_{s, t} \in \mathcal{M}_{\star}^{1}(\mathbb{H})$, the Lévy-measure $\eta$ of $\bar{B}(1)$ is concentrated on $\mathbb{G} \times\{0\} \subseteq \mathbb{H}$, and for nonnegative $g \in \mathcal{C}_{2}(\mathbb{H})$ with $g(e, 0)=0$ we have $\left\langle\bar{B}(1), g \cdot \varphi_{\mathbb{H}}\right\rangle=\left\langle\eta, g \cdot \varphi_{\mathbb{H}}\right\rangle$. Therefore, for $g=h \otimes 1_{\mathbb{R}}$ with $h \in \mathcal{C}_{2}(\mathbb{G})$, we obtain

$$
\left\langle\eta, g \cdot \varphi_{\mathbb{H}}\right\rangle=\lim _{n \rightarrow \infty}\left\langle\sum_{k=1}^{n} \mu^{\bullet}(n, k), h \cdot \varphi_{\mathbb{G}}\right\rangle=\lim _{n \rightarrow \infty}\left\langle\varphi_{\mathbb{G}} \cdot \sum_{k=1}^{n} \mu^{\bullet}(n, k), h\right\rangle,
$$

where $\varphi_{\mathbb{G}} \cdot \mu$ denotes the measure $\nu$ with Radon-Nikodym derivative $\frac{d \nu}{d \mu}=\varphi_{\mathbb{G}}$. Now for any neighbourhood $V$ of $e$ we have $\left.\varphi_{\mathbb{G}}\right|_{\mathbb{C} V} \geq \delta$ for some $\delta>0$. Therefore (4.3) yields weak convergence of the bounded measures $\left.\left.\sum_{k=1}^{n} \mu^{\bullet}(n, k)\right|_{C V} \rightarrow \eta\right|_{C V}$ and by Prohorov's theorem the sequence $\left\{\left.\sum_{k=1}^{n} \mu^{\bullet}(n, k)\right|_{C V}\right\}$ is uniformly tight. Whence, (4.2) follows.

Now we are ready to prove the announced generalized Lie-Trotter formulas that can be seen as weak random integral representations.

Theorem 4.2. With the above notations we have

$$
\bar{\lambda}_{s, t}=\lim _{n \rightarrow \infty} \stackrel{\lfloor n t\rfloor}{\stackrel{*}{*}} T_{-\frac{k-1}{n}}\left(\lambda_{\frac{k-1}{n}, \frac{k}{n}}\right)=\lim _{n \rightarrow \infty} \stackrel{\lfloor n t\rfloor}{\stackrel{\lfloor n s\rfloor+1}{*} \lambda_{k=\lfloor n s\rfloor+1}^{\bullet}} \lambda_{\frac{k-1}{n}, \frac{k}{n}}^{\bullet}
$$

and conversely

$$
\lambda_{s, t}=\lim _{n \rightarrow \infty} \stackrel{\lfloor n t\rfloor}{*} \underset{k=\lfloor n s\rfloor+1}{*} T_{\frac{k-1}{n}}\left(\lambda_{\frac{k-1}{n}, \frac{k}{n}}^{\bullet} .\right.
$$

Proof. According to Siebert [24], 3.6, the conditions (4.1) and (4.2) imply that $\left\{\lambda_{n}(s, t)=*_{k=\lfloor n s\rfloor+1}^{\lfloor n t\rfloor} \lambda_{\frac{k-1}{n}, \frac{k}{n}}^{\bullet}: n \in \mathbb{N}, 0 \leq s \leq t\right\}$ is uniformly tight, hence weakly relatively compact. Let $\left(n^{\prime}\right)$ denote a universal net such that $\lambda_{n}(s, t) \rightarrow \lambda^{*}(s, t)$ along $\left(n^{\prime}\right)$ for all $0 \leq s \leq t$. Then, by Siebert [24], 3.6, $\left(\lambda^{*}(s, t)\right)_{0 \leq s \leq t}$ is a Lipschitz continuous hemigroup with generating functionals $B^{*}(s, t)=\lim _{\left(n^{\prime}\right)} \sum_{k=\lfloor n s\rfloor+1}^{\lfloor n t\rfloor}\left(\lambda_{\frac{k-1}{n}, \frac{k}{n}}^{\bullet}-\varepsilon_{e}\right)$. Hence $B^{*}(s, t)=B^{\bullet}(s, t)$ for all $0 \leq s \leq t$ and, since the hemigroup is uniquely determined by the generating functionals (cf. Siebert [24], 5.7), we have $\lambda^{*}(s, t)=\bar{\lambda}_{s, t}$. The converse limit representation simply follows by $\lambda_{s, t}^{\bullet}=T_{-s} \lambda_{s, t}$. 


\section{REFERENCES}

[1] Becker-Kern, P. (2003) Stable and semistable hemigroups: domains of attraction and selfdecomposability. J. Theoret. Probab. 16 573-598.

[2] Becker-Kern, P. (2004) Random integral representation of operator-semi-selfsimilar processes with independent increments. Stochastic Process. Appl. 109 327-344.

[3] Bogachev, V.I.; Röckner, M.; and Schmuland, B. (1996) Generalized Mehler semigroups and applications. Probab. Theory Relat. Fields 105 193-225.

[4] Dawson, D.A.; and Li, Z. (2006) Skew convolution semigroups and affine Markov processes. Ann. Probab. 34 1103-1142.

[5] Dawson, D.A.; Li, Z.; Schmuland, B.; and Sun, W. (2004) Generalized Mehler semigroups and catalytic branching processes with immigration. Potential Anal. 21 75-97.

[6] Feinsilver, P. (1978) Processes with independent increments on a Lie group. Trans. Amer. Math. Soc. $24273-121$.

[7] Fuhrman, M.; and Röckner, M. (2000) Generalized Mehler semigroups: the non-gaussian case. Potential Anal. 12 1-47.

[8] Hazod, W. (2005) On Mehler semigroups, stable hemigroups and selfdecomposability. In: H. Heyer et al. (eds.) Infinite Dimensional Harmonic Analysis III. Proc. 3rd German-Japanese Symposium, Tübingen, Germany, Sep. 15-20, 2003. World Scientific Publ., pp. 83-97.

[9] Hazod, W.; and Siebert, E. (2001) Stable Probability Measures on Euclidean Spaces and on Locally Compact Groups. Kluwer, Dordrecht.

[10] Hazod, W.; and Scheffler, H.-P. (1999) Strongly $\tau$-decomposable and selfdecomposable laws on simply connected nilpotent Lie groups. Monatshefte Math. 128 269-282.

[11] Heyer, H. (1977) Probability Measures on Locally Compact Groups. Springer, Berlin.

[12] Heyer, H.; and Pap, G. (1997) Convergence of noncommutative triangular arrays of probability measures on a Lie group. J. Theoret. Probab. 10 1003-1052.

[13] Hofmann, K.H.; and Jurek, Z. (1996) Some analytical semigroups occuring in probability theory. J. Theoret. Probab. 9 745-763.

[14] Jeanblanc, M.; Pitman, J.; and Yor, M. (2002) Selfsimilar processes with independent increments associated with Lévy and Bessel processes. Stochastic Process. Appl. 100 223-231.

[15] Jurek, Z.; and Vervaat, W. (1983) An integral representation for self-decomposable Banach space valued random variables. Z. Wahrsch. Verw. Geb. 62 247-262.

[16] Kunita, H. (1997) Stochastic processes with independent increments on a Lie group and their selfsimilar properties. In: I. Csiszár et al. (eds.) Stochastic Differential and Difference Equations. Proceedings Győr, Hungary, Aug. 21-24, 1996. Birkhäuser, Boston. Prog. Syst. Control Theory 23, pp. 183-201.

[17] Lamperti, J. (1962) Semi-stable stochastic processes. Trans. Amer. Math. Soc. 104 62-78.

[18] Li, Z.; and Wang, Z. (2004) Generalized Mehler semigroups and Ornstein-Uhlenbeck processes arising from superprocesses over the real line. Inf. Dim. Anal. Quantum Probab. Relat. Top. 7 591-605.

[19] Maejima, M.; and Naito, Y. (1998) Semi-selfdecomposable distributions and a new class of limit theorems. Probab. Theory Relat. Fields 112 13-31.

[20] Maejima, M.; and Sato, K.I. (2003) Semi-Lévy processes, semi-selfsimilar additive processes, and semi-stationary Ornstein-Uhlenbeck type processes. J. Math. Kyoto Univ. 43 609-639.

[21] Niedbalska-Rajba, T. (1981) On decomposability semigroups on the real line. Colloq. Math. 44 $347-358$.

[22] Schmuland, B.; and Sun, W. (2001) On the equation $\mu_{t+s}=\mu_{s} * T_{s} \mu_{t}$. Statist. Probab. Lett. 52 $183-188$. 
[23] Shah, R. (1996) Limits of commutative triangular systems on real and p-adic groups. Math. Proc. Cambridge Philos. Soc. 120 181-192.

[24] Siebert, E. (1982) Continuous hemigroups of probability measures on a Lie group. In: H. Heyer (ed.) Probability Measures on Groups. Proceedings Oberwolfach, 1981. Springer, Berlin. Lecture Notes Math. 1080, pp. 362-402.

Peter Becker-Kern, Faculty of Mathematics, Dortmund University of Technology, D-44221 Dortmund, Germany

E-mail address: pbk@math.uni-dortmund.de

Wilfried Hazod, Faculty of Mathematics, Dortmund University of Technology, D-44221 Dortmund, Germany

E-mail address: wilfried.hazod@math.uni-dortmund.de 


\section{Preprints ab 2008}

2008-01

2008-02

2008-03

2008-04

2008-05

2008-06

2008-07

2008-08

2008-09

$2008-10$
Henryk Zähle

Weak approximation of SDEs by discrete-time processes

\section{Benjamin Fine, Gerhard Rosenberger}

An Epic Drama: The Development of the Prime Number Theorem

Benjamin Fine, Miriam Hahn, Alexander Hulpke, Volkmar große Rebel, Gerhard Rosenberger, Martin Scheer

All Finite Generalized Tetrahedron Groups

Ben Schweizer

Homogenization of the Prager model in one-dimensional plasticity

Benjamin Fine, Alexei Myasnikov, Gerhard Rosenberger

Generic Subgroups of Group Amalgams

Flavius Guiaş

Generalized Becker-Döring Equations Modeling the Time Evolution of a Process of Preferential Attachment with Fitness

Karl Friedrich Siburg, Pavel A. Stoimenov

A scalar product for copulas

Karl Friedrich Siburg, Pavel A. Stoimenov

A measure of mutual complete dependence

Karl Friedrich Siburg, Pavel A. Stoimenov

Gluing copulas

Peter Becker-Kern, Wilfried Hazod

Mehler hemigroups and embedding of discrete skew convolution semigroups on simply connected nilpotent Lie groups 